\title{
A new organic solvent tolerant protease from Bacillus pumilus 115b
}

\begin{abstract}
Five out of the nine benzene-toulene-ethylbenzene-xylene (BTEX) tolerant bacteria that demonstrated high protease activity on skim milk agar were isolated. Among them, isolate $115 \mathrm{~b}$ identified as Bacillus pumilus exhibited the highest protease production. The protease produced was stable in $25 \%(\mathrm{v} / \mathrm{v})$ benzene and toluene and it was activated 1.7 and 2.5 - fold by $\mathrm{n}$-dodecane and $\mathrm{n}$-tetradecane, respectively. The gene encoding the organic solvent tolerant protease was cloned and its nucleotide sequence determined. Sequence analysis revealed an open reading frame (ORF) of 1,149 bp that encoded a polypeptide of 383 amino acid residues. The polypeptide composed of 29 residues of signal peptide, a propeptide of 79 residues and a mature protein of 275 amino acids with a calculated molecular mass of 27,846 $\mathrm{Da}$. This is the only report available to date on organic solvent tolerant protease from B. pumilus.
\end{abstract}

Keyword: Organic solvent tolerant protease,Cloning,Bacillus pumilus, Isolation 